\title{
Optimization of Dynamic Characteristics for Clapper Automobile Electromagnetic Relay Based PSO
}

\author{
Jifeng Guo, Yuxuan Bi and Honge Ren* \\ Northeast forestry university, Heilongjiang, Harbin, China \\ gjf1000@126,biyuxuan1990@163.com,renhonge163@163.com
}

\begin{abstract}
Electromagnetic system (EMS) is the important part of automobile electromagnetic relay (EMR). A new approach integrating advantages of magnetic equivalent circuit (MEC) method and finite element method (FEM) was proposed to quickly calculate the dynamic characteristics of EMS. The approach is applied in dynamic modeling, design refinement and performance optimization, where repetitive calculation of electromagnetic character is needed. Taking increasing the contacts' breaking velocity and decreasing closing velocity as goals, the EMS was optimized by particle swarm optimization (PSO), and the results indicate the feasibility of the approach.
\end{abstract}

Keywords: Electromagnetic system, dynamic characteristics, magnetic refinement, particle swarm optimization

\section{Introduction}

The clapper type rely is the most widely used electrical switching element in Automobile, its performance, life and reliability directly affect the safe and reliable operation of the Automobile ${ }^{[1-2]}$. MEC and FEM are the general method to the calculation of EMS. MEC can describe the properties of magnetic field with lumped parameter in low computation time, however insensitive to reflect flux leakage and magnetic saturation accurately. FEM has been the most prevalent technique used for magnetic analysis because of its high accuracy. However, in cases repetitive computation is required, even if the parametric model can be accessible, FEM is so time-consuming that often ruled out of consideration. Therefore, high accuracy and low computation time are both desirable but difficult to achieve at the same time. Research has been done to combine both strong points. Moallem used the flux pattern of highly saturated electromagnetic device obtained from FE analysis to modify MEC for more accurate results ${ }^{[3]}$. Chang tried to explore the synergistic interaction of FE method and analytical method based on generalized machine theory to pursue efficiency and accuracy ${ }^{[4]}$. Space Mapping Technique utilized parameter space transformation to achieve a fine model from a course model, usually MEC and other analytical model ${ }^{[5-8]}$. Usually the result from efficient MEC method is complemented by that from accurate FEM.

A new approach based on advantages of MEC and FEM is explored by introducing proportional coefficient matrix, including feature points in different working states obtained from FEM. The approach is based on the following assumptions:

a) Magnetic materials are isotropic and the thermal effect is negligible.

b) Fringing flux that doesn't produce torque can be treated as equivalent leakage flux;

c) In certain working state for electromagnetic devices, the leakage flux and saturation cause mainly differences between MEC and FEM method if the MEC model is fine enough.

\footnotetext{
${ }^{*}$ Corresponding Author
} 


\section{Introduce of Proportional Coefficient Method (PCM)}

Define the ratio of FEM result and MEC result as the proportional coefficient:

$$
M\left(x_{1}, x_{2}, \cdots x_{n}\right)=\frac{f_{F E M}\left(x_{1}, x_{2}, \cdots x_{n}\right)}{f_{M E C}\left(x_{1}, x_{2}, \cdots x_{n}\right)}
$$

Where the $f_{F E M}$ and $f_{M E C}$ are the calculation result (magnetic torque or coil current), $x_{1}, x_{2}, \cdots x_{n}$ are the geometrical dimensions which can affect the calculation result of magnetic system. If we can get these coefficient before the calculation of magnetic dynamic characteristics, the accuracy would be closed to the FEM at the same time the velocity of calculation would be fast only applying the MEC to calculate magnetic system. The function $f_{F E M}\left(x_{1}, x_{2}, \cdots x_{n}\right)$ and $f_{E M C}\left(x_{1}, x_{2}, \cdots x_{n}\right)$ are differentiable in the dimensions range of $x_{1}, x_{2}, \cdots x_{n}$. From formula (1) we can see that the proportional coefficient function $M$ is also differentiable. Therefore, the total differential of $M$ in $\mathrm{N}$ dimensional space point $\left(x_{1}^{*}, x_{2}^{*}, \cdots x_{n}^{*}\right)$ can be described as:

$$
\left.d M\right|_{\left(x_{1}^{*}, x_{2}^{*}, \cdots x_{n}^{*}\right)}=\frac{\partial M}{\partial x_{1}}\left(x_{1}-x_{1}^{*}\right)+\frac{\partial M}{\partial x_{2}}\left(x_{2}-x_{2}^{*}\right)+\cdots+\frac{\partial M}{\partial x_{n}}\left(x_{n}-x_{n}^{*}\right)
$$

Where, the $\frac{\partial M}{\partial x_{1}}, \frac{\partial M}{\partial x_{2}}, \cdots \frac{\partial M}{\partial x_{n}}$ are the partial derivatives of proportional coefficient at the points $\left(x_{1}^{*}, x_{2}^{*}, \cdots x_{n}^{*}\right)$ respectively.

Taking a neighborhood point of the point $\left(x_{1}^{*}, x_{2}^{*}, \cdots x_{n}^{*}\right)$, its partial derivative can be described as:

$$
\left\{\begin{array}{l}
\frac{\partial M}{\partial x_{1}} \approx\left[\mathrm{M}\left(x_{1 \lambda}^{*}, x_{2}^{*}, \cdots x_{n}^{*}\right)-\mathrm{M}\left(x_{1}^{*}, x_{2}^{*}, \cdots x_{n}^{*}\right)\right] /\left(\mathrm{x}_{1 \lambda}-\mathrm{x}_{1}^{*}\right) \\
\frac{\partial M}{\partial x_{2}} \approx\left[\mathrm{M}\left(x_{1}^{*}, x_{2 \lambda}^{*}, \cdots x_{n}^{*}\right)-\mathrm{M}\left(x_{1}^{*}, x_{2}^{*}, \cdots x_{n}^{*}\right)\right] /\left(\mathrm{x}_{2 \lambda}-\mathrm{x}_{2}^{*}\right) \\
\vdots \\
\frac{\partial M}{\partial x_{n}} \approx\left[\mathrm{M}\left(x_{1 \lambda}^{*}, x_{2}^{*}, \cdots x_{n \lambda}^{*}\right)-\mathrm{M}\left(x_{1}^{*}, x_{2}^{*}, \cdots x_{n}^{*}\right)\right] /\left(\mathrm{x}_{n \lambda}-\mathrm{x}_{n}^{*}\right)
\end{array}\right.
$$

Then, the proportional coefficient of any one value point in the neighborhood of $\left(x_{1}^{*}, x_{2}^{*}, \cdots x_{n}^{*}\right)$ can be described as:

$$
M\left(x_{1}^{\prime}, x_{2}^{\prime}, \cdots x_{n}^{\prime}\right)=\mathrm{M}\left(x_{1}^{*}, x_{2}^{*}, \cdots x_{n}^{*}\right)+\left.d M^{\prime}\right|_{\left(x_{1}^{*}, x_{2}^{*}, \cdots x_{n}^{*}\right)}
$$

Where the

$$
\left.d M^{\prime}\right|_{\left(x_{1}^{*}, x_{2}^{*}, \cdots x_{n}^{*}\right)}=\frac{\partial M}{\partial x_{1}}\left(x_{1}^{\prime}-x_{1}^{*}\right)+\frac{\partial M}{\partial x_{2}}\left(x_{2}^{\prime}-x_{2}^{*}\right)+\cdots+\frac{\partial M}{\partial x_{n}}\left(x_{n}^{\prime}-x_{n}^{*}\right)
$$

Therefore the proportional coefficient s of any geometrical dimensions can be obtained through formulas (1) (5). 


\section{Calculation of Dynamic Characteristics Description}

\subsection{Analytical Modeling and Proportional Coefficient}

HELLA/JD1911 automobile relay, a typical rotational electromagnetic system, is chosen as an example to elaborate the research. The magnetic material is DT4E and nominal voltage is $24 \mathrm{~V}$. The turns number of the coil is 1850 and the coil resistance is 90 $\Omega$. Figure1 shows the photo and structure of relay.

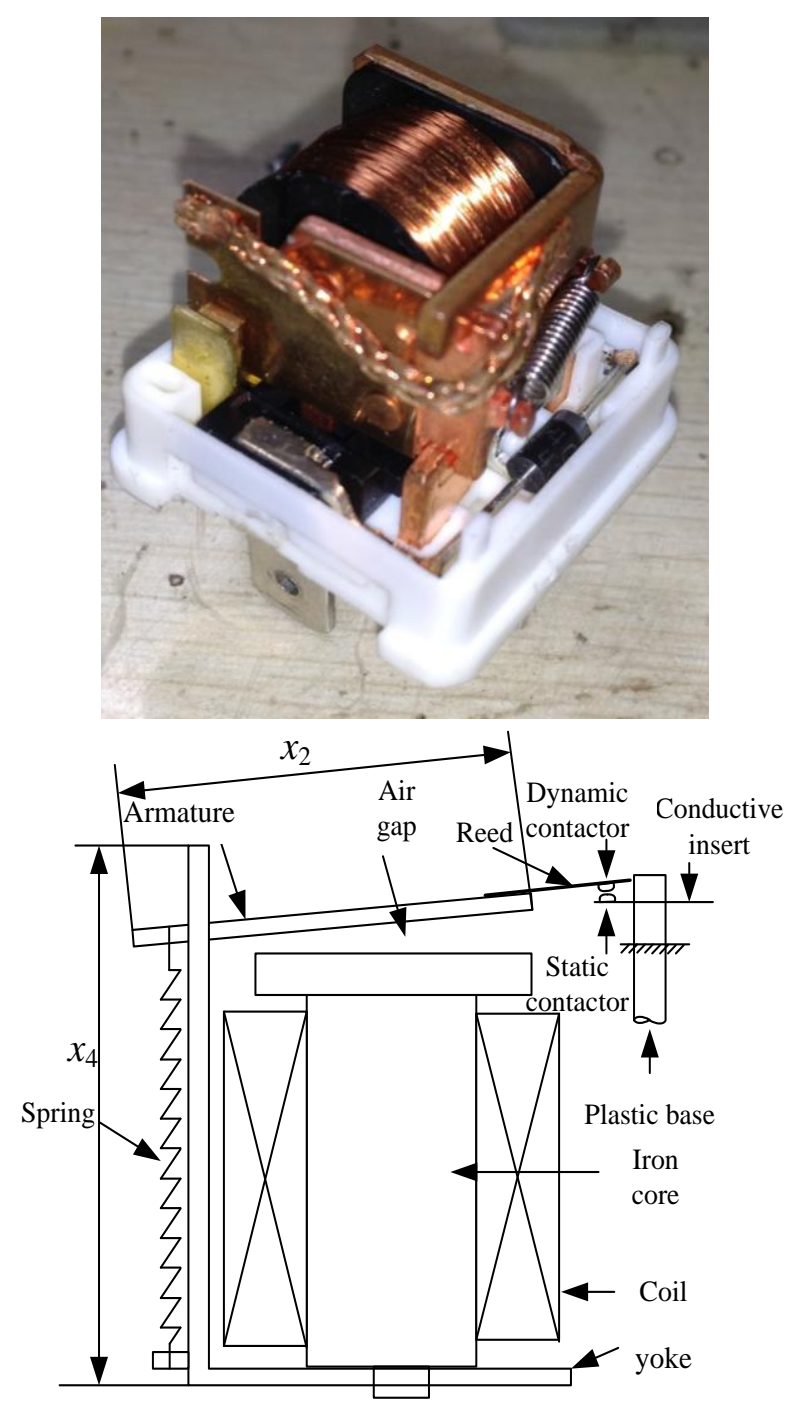

Figure 1. Structure And Equivalent Magnetic Circuit Of Clap-Type Rely

Four key sizes are take as the optimization variable quantity shown in Figure1: x1- the width of armature, $x 2$ - the length of armature, $x 3$ - the width of yoke, $x 4$ - the length of yoke, and their range of values are $[12.6,13.0] \mathrm{mm},[14.0,45.0],[15.7,16.5] \mathrm{mm}$ and[11.3,12.1] $\mathrm{mm}$. In these range of values, the points value for the calculation of proportional coefficient are shown in Table1. In order to realize the fast and refined calculation of every geometry dimensions, in the value range of Table1, respectively building magnetic system model and magnetic circuit model, calculating the proportional coefficient $\mathrm{s}$ is needed. 


\section{Table 1. Feature Points Calculated of Geometry Dimensions Within Their Respective Ranges By FEM}

\begin{tabular}{lll}
\hline $\begin{array}{l}\text { geometry } \\
\text { dimensions }\end{array}$ & $x_{i j}(i=1,2,3,4 ; j=1,2 . \cdots m)$ & $x^{*}$ \\
\hline$x_{1}$ & $12.6,12.7,12.8,12.9,13.0$ & $x_{1}^{*}=12.8 \mathrm{~mm}$ \\
$x_{2}$ & $14.0,14.2,14.6,14.8,15.0$ & $x_{2}^{*}=14.6 \mathrm{~mm}$ \\
$x_{3}$ & $15.7,15.9,16.1,16.3,16.5$ & $x_{3}^{*}=16.1 \mathrm{~mm}$ \\
$x_{4}$ & $11.3,11.5,11.7,11.9,12.1$ & $x_{4}^{*}=11.7 \mathrm{~mm}$ \\
\hline
\end{tabular}

Figure 2 is the equivalent magnetic circuit. $R_{a}, R_{i}, R_{y}, R_{\delta 1}$ and $R_{\delta 2}$ represent the corresponding reluctance and $F_{\text {coil }}$ is the magneto motive of the coil. Actually for static character,

$$
F_{\text {coil }}=N I \quad N=\frac{U}{R}
$$

Where $N, U$ and $R$ are the number of turns, the coil voltage and coil resistance respectively. The equivalent circuit should be as fine as possible so that the error caused by the lumped parameter be reduced to the most. Thus, the compensation of flux leakage and saturation can be more accurate. The reluctance of permeability magnetic material can be usually calculated with relative permeability $\mu_{r}$, length $l$ and sectional area $A$ :

$$
R_{m}=\frac{U_{m}}{\Phi_{m}}=\frac{\int H d l}{\int B d A}=\frac{H l}{B A}=\frac{l}{\mu_{r} A}
$$

Actually, length $l$ can be modified referring to the isovalue results in FE method because $\int H d l$ can be accessible in FE software, as is shown in Figure 3(a). Thus, $l$ can be modified as:

$$
\left\{\begin{array}{l}
R_{m}=\frac{\int H d l}{\int B d A}=\frac{\tilde{l}}{\mu_{r} A} \\
\tilde{l}=\frac{\int H d l}{H_{\max }}
\end{array}\right.
$$

The calculation of gap reluctance also seriously impacts the accuracy of MEC method. It can be obtained by analytical infinitesimal method, the diagram and parameters are shown in Figure 3(b).

$$
\Lambda_{\delta}=\int_{R_{2}}^{R_{1}} \mu_{0} \frac{b}{r \theta} d r=\frac{\mu_{0} b}{\theta} \operatorname{In} \frac{R_{2}}{R_{1}}
$$

Thus the torque-angle and coil magnetic flux (CMF)-angle characteristics of certain coil current can be calculated efficiently by M-programming in matlab.

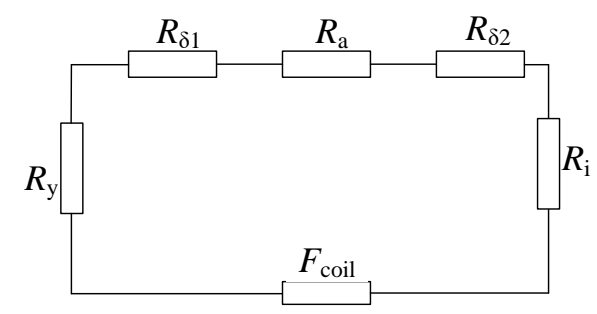

Figure 2. Equivalent Magnetic Circuit 


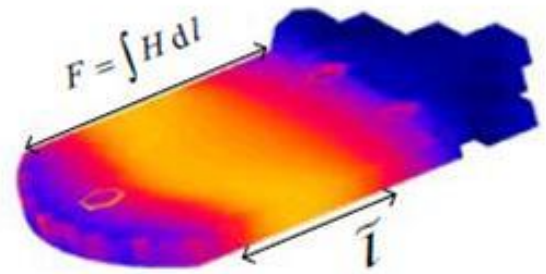

(a) Armature

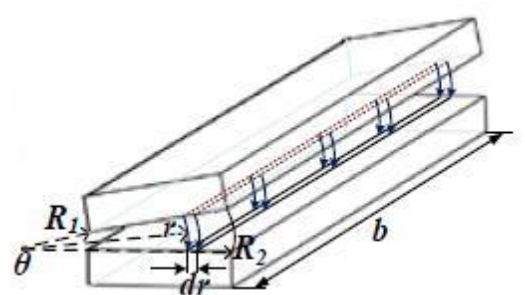

(b) Equivalent Air Gap

Figure 3. Diagram of Analytical Modeling

\subsection{Calculation of Dynamic Characteristic}

HELLA/JD1911 is constitutive of electromagnetic system and mechanical system. To obtain dynamic characteristics, coil flux $\psi(i, \theta)$ and electromagnetic force torque $E T(i, \theta)$ are requisite for electromagnetic system and reaction force torque $R T(i, \theta)$ for mechanical system. The analytical model of mechanical system can be obtained with deformation energy method and calculating $R T(i, \theta)$ is fast and accurate. Then the transient state process can be accessible by solving the following differential equations using 4th order Runge-Kutta method.

$$
\left\{\begin{array}{l}
u=i R+\frac{d \psi(i, \theta)}{d t} \\
E T(i, \theta)-R T(\theta)=J \frac{d^{2} \theta}{d^{2} t}
\end{array}\right.
$$

Where $u$ is the coil voltage, $i$ is the coil current, $R$ is the coil resistance, $t$ is the time, $J$ is the rotational inertial of armature and $\theta$ is the armature position. With the help of proportional coefficient, the calculation process is shown in Figure 4.

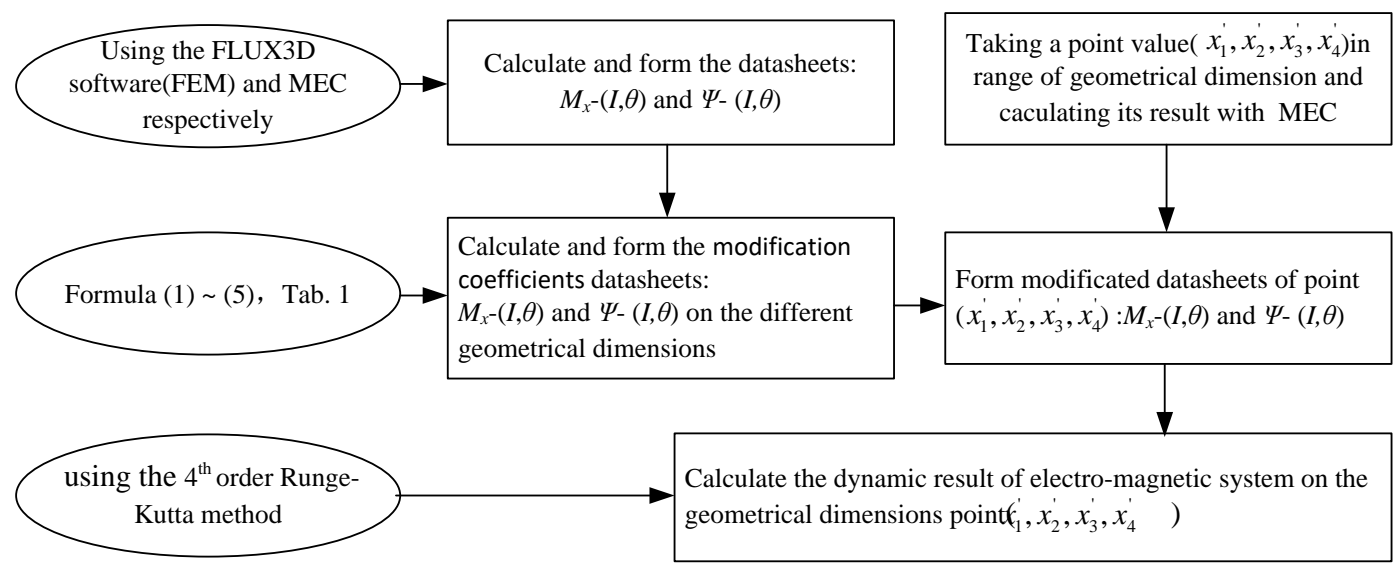

Figure 4. Flow Chart Of Solving Dynamic Characteristics By PCM

\subsection{Comparison of Calculation Result}

In this case, the $\mathrm{x} 1$ is $1.05 \mathrm{~mm}$, the $\mathrm{x} 2$ is $12.45 \mathrm{~mm}$, the $\mathrm{x} 3$ is $1.18 \mathrm{~mm}$ and the $\mathrm{x} 4$ is $1.83 \mathrm{~mm}$. Figure 5 shows the calculation result comparison of the PCM and FEM. From the comparison curves and table data we can see that, the calculation results of two methods are very similar, the most difference is within $3 \%$ and the proportional coefficient method is rather efficiency. 

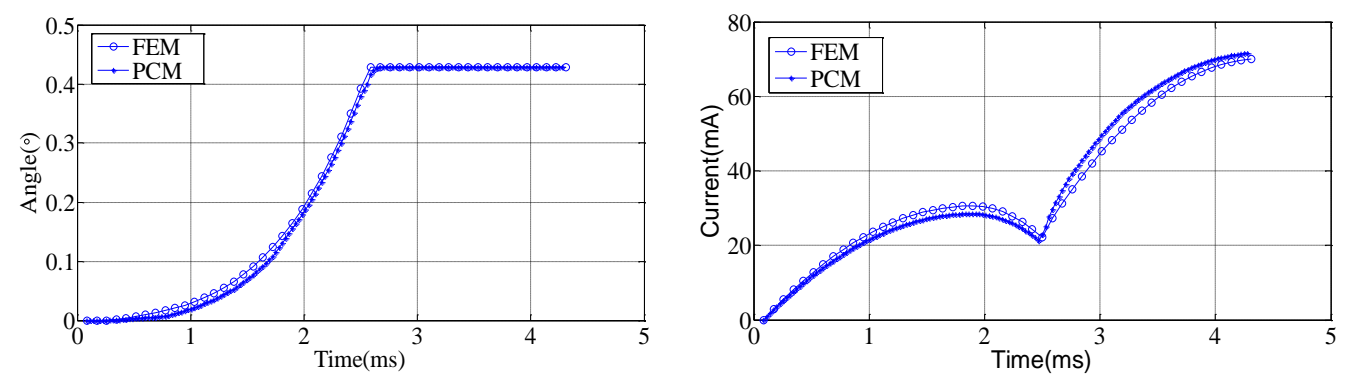

Figure 5. Result Comparison by PCM and FEM

The time consumed by PCM approach is 20-30 second in MATLAB while the FE method over $270 \mathrm{~min}, 50$ time steps computed, the calculation efficiency of PCM is much higher than of FEM. The PCM can efficiently calculate the dynamic characteristics including actuation time, operating voltage, separation velocity, etc. It can describe the dynamic process accurately and efficiently, which makes for dynamic characteristic optimization possible

\section{Optimization Procedure of Magnetic System of Automobile Relay}

Breaking and closing velocity of the contact, which are closely related to contactor's mechanical life-span and arc erosion caused by bounce, are combined as a target to optimize. The Breaking velocity is larger-the-better while the closing velocity is smallerthe-better.

In this paper, the optimization proceeds with particle swarm optimization (PSO). PSO is a global optimization for solving continuous problem. In 1995, Kennedy J. and Eberhart were inspired by the observation of population behavior and put forward this concept for the first time [9]. Particle Swarm optimization is defined by defining a set of random solutions and the corresponding random direction of development, each solution is called an individual, also known as particle. As an evolutionary optimization, iterative computation is accompanied by an update of the solution every single time. The global optimal solution is obtained by the combination of current population optimal solution and particle history optimal solution ${ }^{[10]}$.

In this case, the target of optimization is to combine all design goals into a single function. The task is to solve the problem: Maximum the objection function:

$$
Z\left(x_{1}, x_{2}, \cdots x_{n}\right)=\sigma_{1} \operatorname{Vel}_{b r e}\left(x_{1}, x_{2}, \cdots x_{n}\right)+\sigma_{2} \operatorname{Vel}_{c l o}\left(x_{1}, x_{2}, \cdots x_{n}\right) \rightarrow \operatorname{Max}
$$

Where Velbre is the breaking velocity and Velclo is the closing velocity controlled by input parameter combination $x_{1}, x_{2}, \cdots x_{n}$. Weight coefficient $\sigma_{1}>0$, and the values can be obtained by adaptive weight approach principle.

In this case, the sizes of the particle populations are 30, 80 and 120.The final optimal results are shown in Figure 6. Each optimal execution time is in relation to the size and the algebraic of evolution of particle populations. When the evolution generation is 80 , for 30 particles, calculated in mat lab for 5 hours 12 minutes, for 80 particles, it's 14 hours 20 minutes, for 120 particles, it's 22 hours and 30 minutes. Apparently, with the particles increase, due to the collaborative search, the needed iteration times of evolution to find optimal solution would be reduce. When the number of particles is 80 and 120, the final convergence values of the global optimal solution are almost the same. In order to fully search the solution space, the number of particles generally takes 5-10 times. 


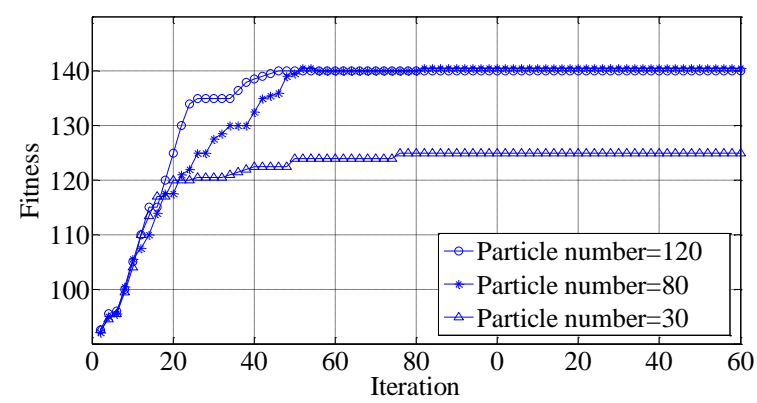

Figure 6. Result Comparison of PSO

Table 4 shows the input and output parameters compassion before optimization and after optimization. After optimization, the breaking velocity increased $3.97 \%$, and the closing velocity dropped $12.03 \%$, which greatly reduced the contact bounce. Clearly, in this case, the optimization result is effective for the increase of life time of contactors.

Table 2. Comparison Of Input And Output Parameter Values Before And After Optimization

\begin{tabular}{|c|c|c|}
\hline Paramaters & before optimization & after optimization \\
\hline $\begin{array}{l}x_{1}, \text { the width of } \\
\text { Armature }(\mathrm{mm})\end{array}$ & 12.8 & 12.9 \\
\hline $\begin{array}{l}x_{2}, \text { the length of } \\
\text { Armature }(\mathrm{mm})\end{array}$ & 14.6 & 14.3 \\
\hline $\begin{array}{l}x_{3}, \text { the width of yoke } \\
(\mathrm{mm})\end{array}$ & 16.1 & 15.9 \\
\hline $\begin{array}{l}x_{4}, \text { the length of yoke } \\
(\mathrm{mm})\end{array}$ & 11.7 & 11.8 \\
\hline $\begin{array}{l}\text { breaking velocity } \\
\operatorname{Vel}_{b r e}(\mathrm{~mm} / \mathrm{s})\end{array}$ & 377 & 392 \\
\hline $\begin{array}{l}\text { closing } \\
\text { velocity } V e l_{c l o}(\mathrm{~mm} / \mathrm{s})\end{array}$ & 806 & 709 \\
\hline
\end{tabular}

\section{Conclusions}

1) A new approach based on MEC and coefficient matrix by some feature points from FE method, which can make dynamic analysis of electromagnetic device have the advantages of efficiency of MEC and precision of FEM, is put forward and elaborated. Coefficient matrix is introduced to calculate dynamic magnetic characteristics of varies geometrical dimension automobile EMR.

2) Taken together the breaking velocity and closing velocity, the objective function is built for the electric contacts life optimization. By some boundary conditions, the optimal solution of the current situation was gotten by using PSO methods which were good at handling high-dimensional complex issues. After optimization, the breaking velocity increased $3.97 \%$, and the closing velocity dropped $12.03 \%$.

3) This research provides the efficiency and feasible method for the other similar problems about electromagnetic system or contact erosion.

\section{Acknowledgments}

This work was supported by the Fundamental Research Funds for the Central Universities (2572014CB27) and the science and technology project of Heilongjiang Province Education Department No. 12533029. 


\section{References}

[1] Y. W and G. Liu, "Thermal Field Simulation Of Miniature Automotive Relays Based On Ansys", J. Electrical \& Energy Management Technology, vol. 2, (2015), pp. 27-31.

[2] X. Li, "Study On Static And Dynamic Based On Virtual Characteristics Of Automobile Relay Prototype Technology", J. Electrical \& Energy Management Technology, 20, (2014), pp. 20-23.

[3] M. Moallem and G. E. Dawson, "An Improved Magnetic Equivalent Circuit Method For Predicting The Characteristics Of Highly Saturated Electromagnetic Devices”, IEEE Trans. Magn., vol. 34, (1998), pp. 3632-3635.

[4] L. Chang, G. E. Dawson and T. R. Eastham, "Combined Finite Element And Analytical Methods For Rotor Design Of Permanent Magnet Synchronous Motors”, Electric Machines And Power Systems, vol. 26, (1998), pp. 465-476.

[5] J. W. Bandler, R. M. Biernacki, S. H. Chen, P. A. Grobelny and R. H. Hemmers, "Space Mapping Technique For Electromagnetic Optimization”, IEEE Trans. Microwave Theory Tech., (1994), vol. 42, no. 12 , pp. 2536-2544.

[6] H.K. Choi, D.H. Kim, H. Park and S.Y. Hahn, “A New Design Technique Of Magnetic Systems Using Space Mapping Algorothm”, IEEE Tran. Magn, (2001), vol. 37, no. 5, pp. 3627-3630.

[7] J.Du, D. Liang and Q. Li, "Magnetic Saturation And End Effects In Lsrms Modelling”, In Compel: The International Journal For Computation And Mathematics In Electrical And Electronic Engineering, (2012), vol. 31, no. 1, pp. 140-153.

[8] K. Hameyer and R. Hanitsch, "Numerical Optimization Of The Electromagnetic Field By Stochastic Search And Mec-Model”, IEEE Trans.Magn., (1994), vol. 30, no. 5, pp. 3431-3434.

[9] J. Kennedy and R.C Eberhart, "Particle Swarm Optimization, Ieee International Conference On Neural Networks”, (1995), no.4,pp,1942-1948.

\section{Authors}

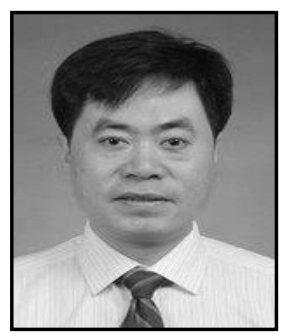

Jifeng Guo was born in 1974. He received the Ph.D. degree from Harbin Institute of Technology, China, in 2011. He is currently an associated professor of information and computer engineering college at school of Northeast forestry university. His main research interests include the reliability theory simulation techniques of Vehicle electrical appliance.

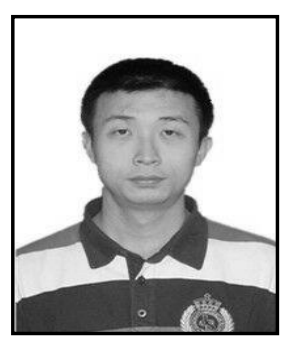

Yuxuan Bi was born in 1990. He received the Bachelor's degree from Northeast Agricultural University, China, in 2013. He is currently reading in Northeast Forestry University. His main research interests include the reliability theory and intelligent control.

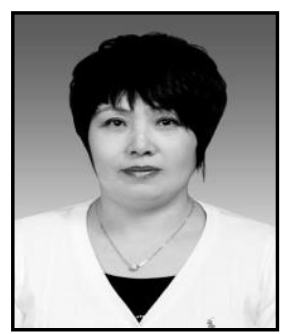

Corresponding author Honge Ren was bornin 1962. She received the Ph.D.degree from Northeast forestry university, China, in 2009. She is currently a professor of information and computer engineering college at school of Northeast forestry university, supervisor of Dr. Her main research interests include the pattern recognition and intelligent control. 